\title{
Untersuchungen über die radiosensible Phase bei der Antikörperbildung*
}

\author{
Von H. St. Stender, D. Strauch und H. Winter ** \\ Aus der Medizinischen- und Nervenklinik (Direktor: Prof. Dr. Dr. H. Bонм), \\ dem Veterinärhygienischen und Tierseuchen-Institut (Direktor: Prof. Dr. E. Roots) \\ und der Strahlenabteilung der Medizinischen- und Nervenklinik (Leiter: Doz. Dr. Th. Hornyкiewytsch) \\ der Justus Liebig-Universität Gießen \\ (Z. Naturforschg. 13 b, 17-19 [1958]; eingegangen am 14. September 1957)
}

\begin{abstract}
Im Vorgang der Antikörperbildung wird von verschiedenen Autoren eine wenige Stdn. dauernde radiosensible Anfangsphase und eine nachfolgende radioresistente Produktionsphase unterschieden. Für die veränderte Strahlenempfindlichkeit ist der Abstand der Bestrahlung vom Zeitpunkt der Antigengabe und der hierdurch eingeleiteten spezifischen Vorgänge verantwortlich gemacht worden. Um die spezielle Bedeutung des Antigens für die unterschiedliche Radiosensibilität zu untersuchen, verabreichten wir bei Ratten kurze Zeit nach der Ganzkörper-Bestrahlung mit $200 \mathrm{r}$ an Stelle des Antigens subkutan eine Kohlesuspension zur Stimulation des lympho-retikulären Gewebes und gaben das Antigen erst $48 \mathrm{Stdn}$. nach der Strahleneinwirkung. Auch unter diesen Bedingungen findet eine zeitgerechte Antikörperbildung statt. In den wenigen Stdn., bevor der Strahlenschaden voll ausgebildet ist, wird durch die Kohlestimulation eine beschleunigte Restitution des lympho-retikulären Gewebes und seiner Funktionsfähigkeit hinsichtlich der Antikörperbildung eingeleitet. Eine von der Gegenwart des Antigens abhängige radiosensible Phase besteht daher nicht.
\end{abstract}

Seit den Untersuchungen von Benjamin und SLukA $^{1}$ lassen sich zwei Arten einer Beeinflussung der Antikörperbildung durch Röntgenstrahlen unterscheiden. Bestrahlungen, die der Antigeninjektion vorausgehen, hemmen die Immunkörper-Produktion erheblich oder unterdrücken sie nach großen Dosen fast vollständig. Die schon angelaufene Antikörperbildung wird dagegen durch die Strahlenwirkung kaum beeinflußt. Über diese allgemeine Zeitabhängigkeit der Strahlenschädigung bei Vor- und Nachbestrahlungen hinaus stellten Taliaferro, Taliaferro und $\mathrm{J}_{\text {anssen }}{ }^{2}$ sowie Dixon, Talmage und Maurer ${ }^{3}$ fest, daß eine gute Antikörperbildung noch stattfindet, wenn die Antigeninjektion dem Strahleninsult in den ersten Stdn. folgt. Aus dieser unterschiedlichen Strahlenempfindlichkeit des Vorganges der Antikörperbildung leiten Dixon und Mitarbb. die Existenz einer kurzen radiosensiblen Anfangsphase und einer anschließenden weitgehend radioresistenten Produktionsphase ab. Nur wenn die Strahlenschädigung zunächst noch nicht voll ausgebildet ist, kann nach Ansicht der genannten Autoren ,an adaptation of the globulin synthesis to the new antigen" noch stattfinden. Ist der strahlenemp-

* Die Untersuchungen wurden durch Mittel der LVA Hessen unterstützt.

** Siehe S. 19.

1 E. Benjamin u. E. Sluka, Wiener Klin. Wschr. 21, 311 [1908].

2 W. H. Taliaferro, L. G. Taliaferro u. E. F. Janssen, J. infect. Diseases 91, 105 [1952]; 94, 134 [1954].

3 F. J. Dixon, D. W. Talmage u. P. H. Maurer, J. Immunology 68, 693 [1952]. findliche Vorgang der Adaptation aber abgeschlossen, dann wird die Produktion der Antikörper durch die Strahleneinwirkung kaum noch behindert. Während Dixon, Talmage und Maurer diesen biochemischen Vorgang als besonders strahlenempfindlich ansehen, suchen Taliaferro, Taliaferro und JANSSEN die Ursache in einer Lokalisationsstörung des Antigens in den zur Antikörperbildung fähigen Zellen oder in einer Störung des frühen Antigenstoffwechsels. Auch $\mathrm{KoHN}^{4}$ zieht die Möglichkeit einer gestörten Antigenfixation in Erwägung.

Allen diesen Deutungen der verschieden großen Strahlenempfindlichkeit im Vorgang der Antikörperbildung ist gemeinsam, daß dem Antigen eine entscheidende Bedeutung zugesprochen wird und die Radiosensibilität eine direkte Abhängigkeit vom Antigen besitzen soll.

Um die Bedeutung der Gegenwart des Antigens und der dadurch eingeleiteten spezifischen Prozesse für die Strahlenempfindlichkeit zu untersuchen, verabreichten wir eine Stde. nach der Bestrahlung an Stelle des Antigens eine subkutane Injektion einer 2-proz. Kohlesuspension und gaben das Antigen erst 48 Stdn. später. Aus den cytologischen Untersuchun-

4 H. J. Koнs, J. Immunology 66, 525 [1951].

5 K. Betke, L. Bickhoff, E. Kammüller u. F. Helpenstein, Klin. Wschr. 33, 619 [1955].

* Anschriften der Verfasser: Dr. H. St. Stender, Strahlenklinik der Universität Marburo Dr. D. Strauch, Veterinärhygienisches und Tierseucheninstitut der Justus-Liebig-

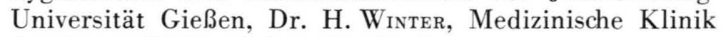
der Justus-Liebig-Universität Gießen. 


\begin{tabular}{|c|c|c|c|c|c|c|c|c|}
\hline \multirow{3}{*}{ Versuchsbedingungen } & \multirow{3}{*}{ [Stdn.] } & \multirow{3}{*}{$\begin{array}{c}\text { Zahl } \\
\text { der } \\
\text { Tiere }\end{array}$} & \multicolumn{6}{|c|}{ Serumantikörper-Titer } \\
\hline & & & \multicolumn{2}{|c|}{ 4. Tag } & \multicolumn{2}{|c|}{ 7. Tag } & \multicolumn{2}{|c|}{ 12. Tag } \\
\hline & & & & $\cdot 10^{-3}$ & & $\cdot 10^{-3}$ & & $\cdot 10^{-3}$ \\
\hline Immunisierung & & 60 & $1: 70$ & $14,4 \pm 0,9$ & $1: 260$ & $3,9 \pm 0,38$ & $1: 180$ & $5,4 \pm 0,9$ \\
\hline $\begin{array}{l}\text { Immunisierung } \\
\text { Bestrahlung nach }\end{array}$ & 48 & 30 & $1: 70$ & $15,2 \pm 0,6$ & $1: 220$ & $4,6 \pm 0,56$ & $1: 160$ & $6,4 \pm 0,57$ \\
\hline $\begin{array}{l}\text { Vorbestrahlung } \\
\text { Immunisierung nach }\end{array}$ & 48 & 42 & $\varnothing$ & $\varnothing$ & $\varnothing$ & $\varnothing$ & $1: 40$ & $27,1 \pm 3,2$ \\
\hline $\begin{array}{l}\text { Vorbestrahlung } \\
\text { Immunisierung und } \\
\text { Kohlesuspension nach }\end{array}$ & 48 & 30 & $\varnothing$ & $\varnothing$ & $\varnothing$ & $\varnothing$ & $1: 60$ & $16,4 \pm 1,8$ \\
\hline $\begin{array}{l}\text { Vorbestrahlung } \\
\text { Immunisierung nach }\end{array}$ & 1 & 30 & $1: 20$ & $63,8 \pm 6,0$ & $1: 120$ & $8,1 \pm 1,0$ & $1: 150$ & $6,9 \pm 0,6$ \\
\hline $\begin{array}{l}\text { Vorbestrahlung } \\
\text { Kohlesuspension nach } \\
\text { Immunisierung nach }\end{array}$ & $\begin{array}{r}1 \\
48\end{array}$ & 32 & $1: 70$ & $14,6 \pm 1,4$ & $1: 170$ & $6,0 \pm 0,5$ & $1: 150$ & $6,8 \pm 0,7$ \\
\hline
\end{tabular}

Tab. 1. Verhalten der Serumantikörper unter verschiedenen Versuchsbedingungen. Mittelwerte der Endtiter und mittlerer Fehler des Mittelwertes in Dezimalbrüchen.

gen von Betke, Bickhoff, Kammüller und HelpenSTEIN ${ }^{5}$ sowie aus eigenen Beobachtungen, wissen wir, daß eine Kohleinjektion zu einer unspezifischen Stimulierung und Aktivierung des lympho-retikulären Gewebes führt, in dem die Antikörperbildung stattfindet.

\section{Methodik}

Versuchstiere: Weibliche Ratten mit einem Gewicht von 120 bis $150 \mathrm{~g}$.

Als Antigen verwandten wir eine Suspension von Brucella melitensis mit einem Gehalt von $3 \cdot 10^{11}$ abgetöteten Keimen in $1 \mathrm{~cm}^{3}$. Hiervon wurden $0,2 \mathrm{~cm}^{3}$ subkutan an der Innenseite des linken Schenkels injiziert.

Die Serumantikörper wurden in absteigenden Verdünnungsstufen mit der Langsamagglutination bestimmt. Als Endtiter wurde die Verdünnungsstufe mit der Agglutination von 50\% und mehr angesehen. In den verschiedenen Versuchsgruppen ist der Mittelwert der Endtiter und ihr mittlerer Fehler in Form der Dezimalbrüche errechnet.

Untersuchungszeitpunkt: 4., 7. und 12. Tag nach Immunisierung.

Zur unspezifischen Stimulation diente uns eine 2-proz. Aufschwemmung von Carbo medicinalis in doppelt dest. Wasser. Hiervon spritzten wir $0,2 \mathrm{~cm}^{3}$ subkutan an der Innenseite des linken Oberschenkels.

Die Ganzkörper-Bestrahlung wurde durchgeführt bei: $190 \mathrm{kV}, 20 \mathrm{~mA}$, HWS $1,1 \mathrm{Cu}, 60 \mathrm{r} / \mathrm{min}$, FTA $60 \mathrm{~cm}$. Dosis: $200 \mathrm{r}$.

Die cytologischen Untersuchungen wurden an Tupfund Ausstrichpräparaten der Leisten-Lymphknoten beider Seiten und der Milz durchgeführt.

Die Ergebnisse sind in Tab. 1 zusammengefaßt.

\section{Ergebnisse}

Die aufgeführten Untersuchungen an Ratten bestätigen die Feststellung, daß die schon im Gang befindliche Antikörperproduktion durch die nachfolgende Strahleneinwirkung (200r) nicht wesentlich behindert wird. Auch wenn die Antigeninjektion innerhalb weniger Stdn. der Bestrahlung folgt, läuft die Immunkörperbildung noch zeitgerecht an. Dabei liegen die Serumtiter zunächst niedriger. Geht die Strahleneinwirkung der Immunisierung um 48 Stdn. voraus, dann wird das Anlaufen der Antikörperbildung wesentlich verzögert und die Antikörpermenge während des Zeitraums der Untersuchung stark verringert. Auch eine zusätzliche Kohleinjektion gemeinsam mit dem Antigen $48 \mathrm{Stdn}$. nach der Bestrahlung ändert diese starke Verzögerung nicht. Ersetzen wir nun aber die Antigengabe kurze Zeit nach der Bestrahlung mit $200 \mathrm{r}$ durch die Injektion einer Kohlesuspension und lassen die Berührung mit dem Antigen erst nach $48 \mathrm{Stdn}$. erfolgen, so findet auch unter diesen Bedingungen eine zeitgerechte kräftige Antikörperbildung statt, obwohl die Zeitspanne zwischen Strahleninsult und Antigeninjektion die gleiche ist, wie bei den Tieren der Vorbestrahlungsgruppe. Die Gegenwart des Antigens vor oder kurze Zeit nach der Bestrahlung hat für die unterschiedliche Strahlenempfindlichkeit daher keine grundsätzliche Bedeutung. Eine spezifische radiosensible Phase, wie sie von Dixon, Talmage und Maurer sowie Talia- 
Ferro, Taliaferro und Janssen herausgestellt ist, besteht auf Grund unserer Beobachtungen nicht.

Der Vergleich der serologischen Ergebnisse mit den cytologischen Befunden, über die an anderer Stelle ausführlich berichtet werden soll, zeigt, daß die unterschiedliche Radioresistenz ihre Ursache im Verhalten der Zellen hat, die zur Antikörperbildung fähig sind. Auch von Fitch, Wissler, La $V_{\text {IA }}$ und BARKER ${ }^{6}$ wird die Bedeutung des zellulären Substrates bei diesem Vorgang hervorgehoben. Wird eine Stimulation des lympho-retikulären Gewebes kurze Zeit nach der Bestrahlung, wenn die Strahlenschädigung noch nicht voll ausgebildet ist, durch ein Antigen oder durch eine Kohlesuspension angeregt, so erholt sich dieses Gewebe schneller vom Strahleninsult. Die Entwicklung, Teilung sowie Transformation der Retikulumzellen und die Bildung von Plasma- zellen ist dann weniger behindert und die Antikörperbildung kann zeitgerecht anlaufen. Auch im lympho-retikulären Gewebe, das 2 Tage vor der Bestrahlung durch ein Antigen aktiviert wurde, sind die antikörperbildenden Zellen den Strahlen gegenüber resistenter. Die Strahlenwirkung an den Zellen, die zur Immunkörperproduktion fähig sind oder schon Antikörper bilden, hängt ab von ihrem $\mathrm{Zu}$ stand im Zeitpunkt der Bestrahlung und von den Eingriffen, die die Manifestation der Schädigung beeinflussen und die Erholung fördern. Die zellulären Vorgänge, die die verschiedene Strahlenempfindlichkeit bei der Antikörperbildung bestimmen, sind unabhängig von der Gegenwart des Antigens und den von ihm eingeleiteten spezifischen biochemischen Prozessen.

\title{
Uber die Korrelation zwischen Partikellänge und Infektiosität beim Vergilbungsvirus der Rüben*
}

\author{
Von KarL-Wolfgang Mundry ** \\ Aus dem Institut für landwirtschaftliche Technologie und Zuckerindustrie an der Technischen \\ Hochschule Braunschweig \\ (Z. Naturforschg. 13 b, 19-27 [1958]; eingegangen am 9. September 1957)
}

\begin{abstract}
Crude leaf sap of plants suffering from sugar beet yellows was partially purified by density gradient centrifugation in the cold at different values of $p_{\mathrm{H}}(6,2-9,0)$. After standing over night at $2^{\circ} \mathrm{C}$ the material was dialysed against buffer of $p_{\mathrm{H}} 6,8$ at room temperature. - As estimated by electron microscopy the most frequent length of the threadlike particles (Fig. 2) which were believed to be the infectious agent of sugar beet yellows shows a gradual shift from longer to shorter ones with rising $p_{\mathrm{H}}(\mathrm{Fig} .1 \mathrm{a}-\mathrm{e})$. The infectivity of the suspensions depends upon the $p_{\mathrm{H}}$ of the preparation too. From the distribution curve of the particle lengths the relative concentrations of particles with different given minimal lengths were estimated. The dependence from the $p_{\mathrm{H}}$ of the preparation of these relative concentrations was compared with that of the infectivity. Best agreement was observed with particles of $12700-12800 \AA$ minimal length (Fig. 3). This length seems to be identical with the "normal length" of the particles found in exsudates (12 400-12500 ̊). From these results it was concluded that a) particles which possess the normal length as found in exsudates are the infective entities of this disease, b) shorter particles are non-infectious, c) the infectious entities of the sugar beet yellows virus are of the threadlike type. There is no evidence that spherical particles are necessary for starting an infection with the sugar beet yellows virus.
\end{abstract}

Viruspartikel besitzen ganz allgemein eine bestimmte Größe und Form. Dies ist besonders auffällig bei stab- und fadenförmigen Virusarten, bei denen nach Vermessung elektronenmikroskopischer Abbildungen die überragende Häufigkeit von Par-

\footnotetext{
* Mit Unterstützung durch die Deutsche For. $\mathrm{schungsgemeinschaft.}$
}

tikeln bestimmter Größe durch die große Häufigkeit einer bestimmten Partikellänge zu erkennen ist (Abb. lf). Diese häufigste Länge, auch „Normal. länge“ genannt, ist für eine gegebene Virusart cha. rakteristisch. Sie beträgt z. B. beim Tabakmosaik.

\footnotetext{
** Neue Adresse: MPI für Biologie, Abt. Melchers, Correns-
} straße 41, Tübingen. 